Research Article

www.jestr.org

\title{
Ability of OFDMA in Handling Interference of Femto Cells Under Random Access Process
}

\author{
Seetaiah Kilaru
}

KL University, India

Received 11 April 2014; Accepted 14 July 2014

\begin{abstract}
The smart phones and other high end application devices opened gates for the challenge of new era of data services. The availability of $4 \mathrm{G}$ spectrum is the main reason behind it. $4 \mathrm{G}$ network architectures concentrated on heterogeneous structures which can handle different cell sizes. To increase the capacity of network in hotspots like inside building or complex, there is need to deploy small cell base stations. These cells are basically not manageable properly due to their ad hoc property. Hence, analysing the nature and interference phenomenon between cells is necessary task. Femto cells are increasing their number from time to time. They are operated under small load and will serve for limited number of users. This paper completely concentrated on the interference issue in Femto cells with respect to multiple access approach. This paper highlighted the ability of OFDMA in handling such interference issues and explained under random access process. LTE stack protocol is also proposed to design MAC algorithm in the context of OFDMA cells.
\end{abstract}

Keywords: OFDMA, Femto Cells

\section{Introduction}

With the technology growth it is observed that, the smart mobiles are in a position to access more traffic particularly in broadband applications. We are estimating that, this ease of access may cross the limit of defined width of broadband. There is a need to change the network designing principles. Temporal and spatial spectral techniques may show a path to solve this problem temporarily. These procedures may not give permanent and efficient solution to reach the necessary broadband limit. Implementation of temporal approach is always effective in $4 \mathrm{G}$ deployment. In India, $4 \mathrm{G}$ systems were not completely established due to various reasons. LTE releases/versions define the respective uplink/downlink speeds. The existed version of release 8 will give $170 / 300 \mathrm{Mbps}$ for uplink/downlink. There are various radio access methods are available in LTE, but Orthogonal Frequency Division Multiple Access (OFDMA) is the key method due to its nature of efficient modulation phenomenon. OFDMA is always recommendable for the problems of multiple interference analysis. One of the added advantages of this method is low complexity in implementation. The efficiency of the OFDMA depends on the factor of diversity [1]. The factor of Quality of Service (QOS) depends on allotment of Time-Frequency domain allocation. This OFDMA technique follows the principle of simultaneous low data rate transmission from multiple users [4].

To achieve over all higher gain and to increase the coverage efficiency, $4 \mathrm{G}$ cells incorporates different cell

\footnotetext{
*E-mail address: dr.seetaiah@gmail.com

ISSN: 1791-2377@ 2014 Kavala Institute of Technology. All rights reserved.
}

structures [10]. The main phenomenon may depend on macro cell which are covering huge area. Along with this, installation of micro cell is also essential in some cases viz. inside big buildings and apartments [3]. Femto cell is also one of the types of macro cell. These small cells in general will cover less distance preferably vary from 10 meters to 100 meters. In telecommunication it is possible to define intermediate link between core network and sub network with the aid of backhaul. These small or Pico cells were best suitable in high density traffic applications. Femto cell is a low power cell/base station which serves for limited area. DSL/Cable is used in Femto cell to connect to the network provider. The world leading operators like Vodafone, orange etc. are already launched this service. The Femto cell and outdoor macro cell run with respect to same spectrum. Interestingly, the typical macro cell is capable to produce very high data rates when compared with the macro cell even both are operated under same spectrum.

Now, LTE or 4G technology along with Femto cell concept will increase the capacity of the network in handling the traffic with excellent data rates. This assumption is only true, if the designer eliminates the inter cell interference factor. Link adaption and diversity issues are very easy to resolve when compared to the interference factor. Cochannel interference parameter is the major concern. This may exist in two forms viz. Femto to Femto interference or base macro to Femto cell. Both of these effects should be avoided to get high Signal to Noise Ratio (SNR) [2, 7,8]. From the parameters of condition of channel, power transmission and method of multiplexing, we can predict downlink interference factor in accurate way. In current era, sharing spectrum issue between Femto and macro cell analysis is a challenging task. Femto cell was basically adhoc in nature. Even though the designer allotted the different 
frequency spectrum, due to its random nature, interference problem is arising in these cells. The absence of macro layer between Femto cells further reduces the accuracy. This paper completely focussed on the issue of Femto to Femto interference. The fig 1 shows the approximated Femto cell network architecture [7].

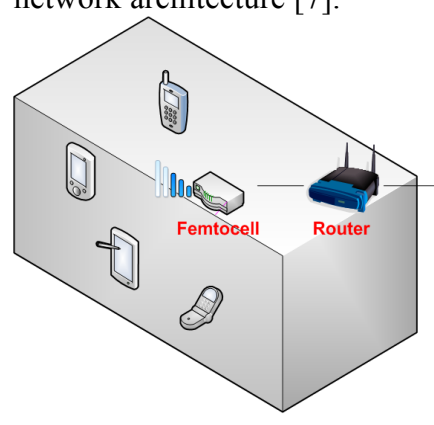

Fig. 1. Femto cell network architecture

The following are the some of the problems while dealing with design and installation of Femto cells. They are

1. Perfect interference coordination is highly impossible between base cell (macro) and defined Femto cell [6].

2. Perfect planning of allocation of frequency for particular Femto cell is not possible. The main reason behind that is users may deploy anywhere in their location.

3. The parameter of interference is dynamic in nature. It completely depends on the user hands and their activities.

There are two ways to analyse the parameters of interference in Femto cells. They are power based method and orthogonal time-frequency allocation of resources [10]. From the survey, it is clear that the most Femto cells operating under $3 \mathrm{G}$ technologies are using power control as a main tool. All existed and proposed systems initially assume that the load of Femto cell is huge [15]. But, in practice it is not true. The designs are ignoring the factor of user activity. Hence, the existed systems are not giving proper accuracy. This paper is completely focussing on the random nature of user activity by defining proper traffic model.

\section{Multiple access techniques in Femto cells based on Orthogonal Frequency Division}

In current scenario, we are using random access technique in the first stage only. In this stage, the main focus is put on requesting a channel for communication between macro and Femto cells and also synchronisation between them. Once the synchronisation was finished, then OFDMA perform remaining action in LTE [15]. LTE in this process is using Reservation Aloha mechanism. That means it reserve the particular bandwidth to the designed system. This will not relate to the original information transmission. That means that no actual information is floating over this Random access channel. Macro cell is covering a huge area and also huge traffic density, so that it should always be active. If the user is successfully admitted by the base station in defined macro cell, the uplink and downlink transmissions scheduled according to the requirement. In case of Femto cell, the story is different because it serves the smaller area. In that area, in general there are less number of users are in active position [11]. Form this assumption; it is clear that most of the Femto cells operated under low duty cycles [7].

From the condition of existence of many number of Femto cell operating under same spectrum is a critical task [9]. Because, transmit-receive pairs basically competing with each other to occupy a channel with proper uplink/downlink transmissions. In this scenario, random access is preferable than conventional access method [2].

Fig 2 represents LTE protocol stack. We are using the flexibility and vulnerability of OFDMA in designing of Femto cell. The time frequency grid of OFDMA will use slotted multi-channel phenomena. Construct the main resource block frame from the grouping concept. Group all possible and existed sub carriers and finally get the main block of frequencies. Then, calculate the channel bandwidth and divide it equally that can be assessed on slot by slot basis. Apply multi-channel multiple access approach to all resource blocks. In this method the user will use only one channel from all possible channels and send information in form of packets through that channel.

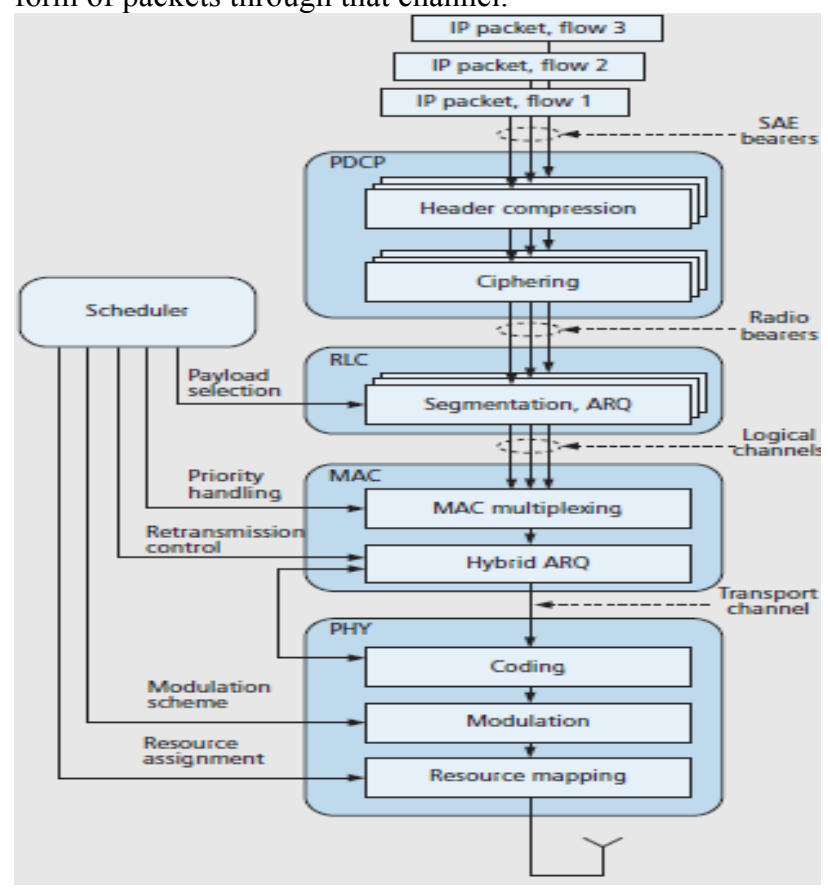

Fig 2. LTE Protocol stack

Existed multi-channel access techniques were inefficient while dealing with the random multiple access system. OFDMA has its own features which are differ from conventional methods. They are

1. Instantaneous channel access facility is always open in OFDMA. In conventional access method, it will take considerable time frame to switch from one channel to another channel.

2. Simultaneous transmission and reception is excellent over available channels with single radio.

In OFDMA Aloha, the factor of try limit is high. In this process, like traditional aloha, it can't wait defined time for retransmission [4,5]. It chooses another sub channel to transmit immediately. The advancement of this OFDMA 
based aloha leads to invention of OFDMA based CSMA/CS. In this CSMA/CS method, the transmission node senses all possibilities of idle channels. From all available sub channels, the user node will select one channel with back off principle of Collision Avoidance [12]. These two methods were excellent when the number of users is preferably more than the number of channels.

In Femto cell, the consideration is completely different from original scenario. In this cell, the number of available sub channels is considerably more than the number of users. The number of available channels is always high, hence to utilise maximum, the user packet data transmitted through number of channels simultaneously.

\section{Advanced protocol stack model}

The transmission of information is done by packets. These IP packets don't enter into the wireless medium directly. They have to travel through the four layers, processed and finalised packets enter into the transmission medium. Compression is the responsibility of Data Convergence protocol (DCP) $[6,14]$. The compressed packets are given to Radio Link Control (RLC) layer. This layer will take packets and then perform the segmentation process; the size of the segmentation will vary from time to time/ slot by slot. This variation depends on MAC layer which gives the information about Resource allocation. The data flow of these packets is shown in fig 3 .

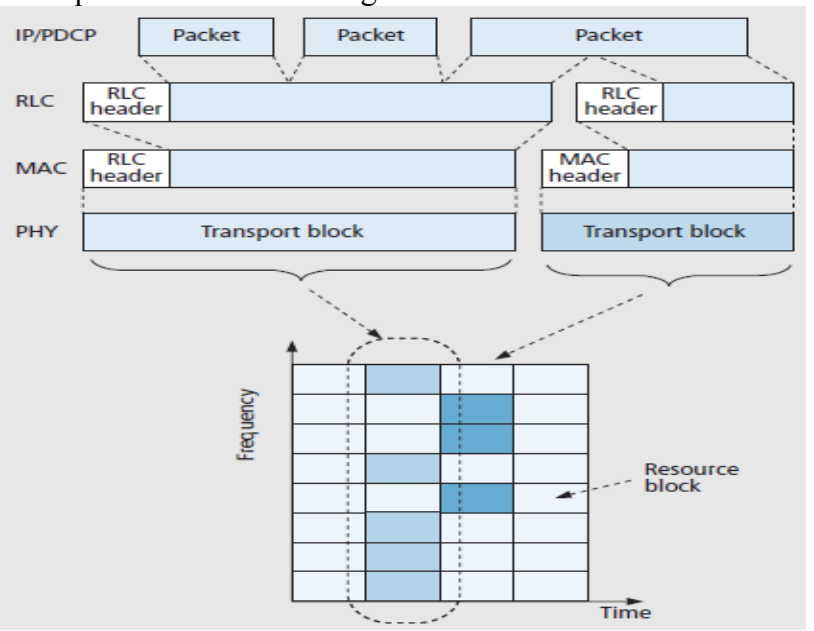

Fig 3. Data flow in stack model

The resource blocks meant for number of available sub channels. Modulation and Coding scheme (MCS) plays vital role in MAC layer. MAC layer always reschedules the information about the availability of number of channels for next transmission. This layer is also responsible for the selection of MCS. This information is passed to the receiver. Based on Resource block and MCS, the size of the required Transport Block (TB) was defined. The customized RLC layer is divided into different PDU's. The size of this Protocol Data Unit (PDU) is the addition of TB and MAC sizes. The MAC layer adds header and creates new TB for Hybrid Automatic Request (H-ARQ) process [5]. MAC layer assigns the sub carriers. Physical layer will do mapping of modulation symbols to the assigned sub carries. The decoding at the receiver is always a challenging task. The delivering of IP packets to the receiver is depending on the TB decoding process. If TB is not decoded properly, then IP packets delivers wrong message or reach the incorrect destination. If TB is not decoded properly, then the H-ARQ will retransmit the required TB. The major drawback of $\mathrm{H}-$ ARQ is, it is not defined to handle errors which cause from strong collisions.

\section{Experimental model}

Here, the two access schemes are tested by considering the Femto cell with low traffic load. The Femto cell is working based on the principle of OFDMA. Let, the OFDMA operated with $\mathrm{N}$ Femto cells and $\mathrm{K}$ sub channels. Each channel is in a position to transmit only one packet in their one of the available sub channels. This channel should maintain in the domain of single collision. The probability of the packet arrive at the user is considered as $\alpha$ and packets are entered based on the Bernoulli arrival process. Now, apply bulk access method, so that the packets are transmitted as a group will occupy all available channels for one slot.

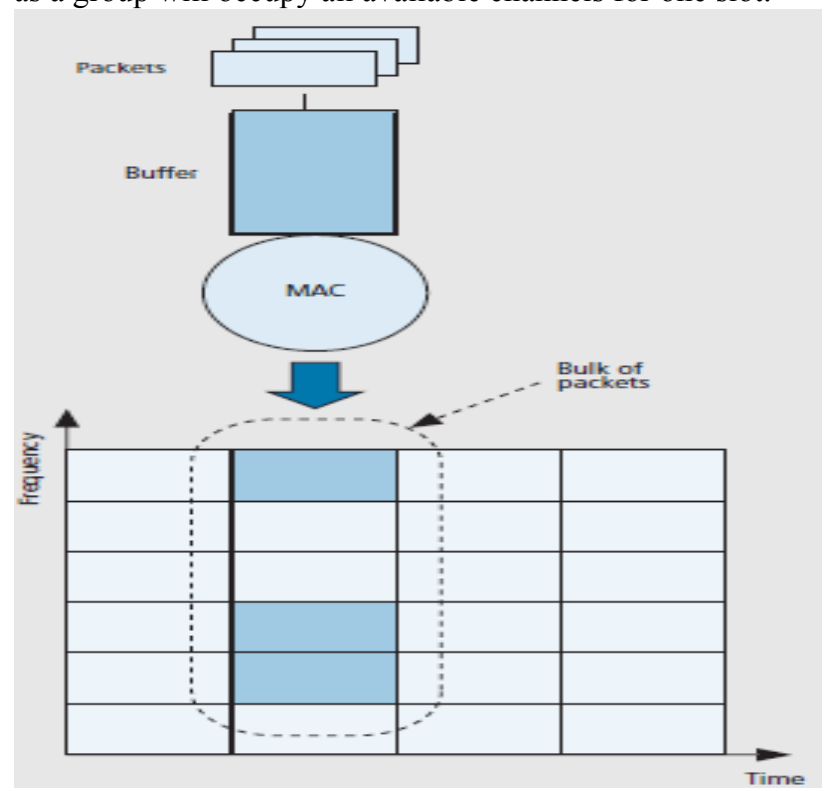

Fig 4. Bulk access model for Femto cell

Femto cells are operated for low traffic application, hence there is number of possibilities are there for the selection of required channels $\mathrm{M}$ out of available $\mathrm{K}$ channels and is observed from fig 4 . Reduction of collision possibility is excellent in selection of random channels.

Consider 12 Femto cells with single user access and $\mathrm{K}=32$ sub channels. We simulated simple aloha and $\mathrm{K}$ aloha with the probability of transmission $1 / \mathrm{N}$. in simple aloha, the bulk is always mapped to sub channel indexed 1 through $\mathrm{M}$. in $\mathrm{K}$ aloha; randomly we selected $\mathrm{M}$ channels out of $\mathrm{K}$ channels. The load of the traffic load is increased from time to time. The fig shows the time required to transmit data successfully with less possibility of collision. In low load region, it clearly indicates that there is a significant reduction in service time. Even though this optimization is not up to the mark, but it really shows a way to access bulk models. From this it is clear that random multiple access phenomenons are possible in OFDMA based Femto cells. 


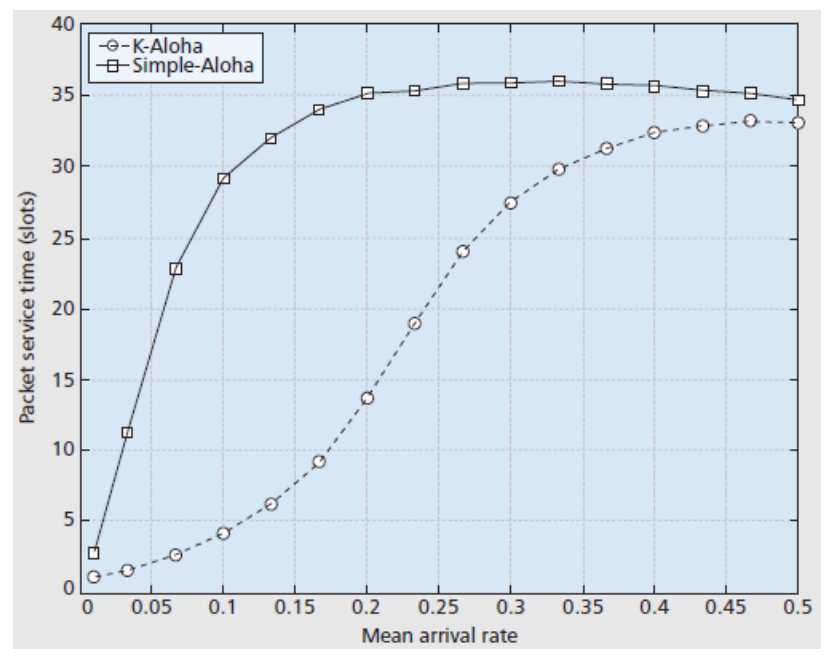

Fig 5. Packet service time K Vs normal Aloha

\section{Conclusion.}

Interference problem in Femto is addressed with the help of OFDMA. The feature of broad bandwidth is one of the main reasons in reduction of interference. This report discussed this issue with traditional multiple access phenomenon. OFDMA based MAC utilises frequency dimension in more suitable way than MAC in normal wireless LAN. Multiple sources is operated with different packets are transmitted over multiple sub channels will reduce the interference problem. This work may enhance the random access MAC protocol implementation in OFDMA Femto cells.

\section{References}

1. Qualcomm, The 1000x Data Challenge, Oct. 2012; $\mathrm{http} / /$ www.qualcomm.com/solutions/wireless-networks/ technologies/1000x-data.

2. M. Nohrborg, "LTE Overview," 3GPP; http://www.3gpp.org/LTE, Aug. 2012.

3. Small Cell Forum Ltd. "Small Cells — What's the Big Idea?" Feb. 2012; http://www.smallcellforum.org.

4. T. Zahir et al., "Interference Management in Femtocells," IEEE Commun. Surveys \& Tutorials, vol. PP, no. 99, 2012, pp. 1-19.

5. "Residential Femtocells," Aug. 2012; http://www.ubiquisys.com/small-cells-femtocells-residential.

6. G. Fodor et al., "Intercell Interference Coordination in OFDMA Networks and in the 3GPP Long Term Evolution System," $J$. Commun., vol. 4, no. 7, Aug. 2009.

7. G. Boudreau et al., "Interference Coordination and Cancellation for 4G Networks," IEEE Commun. Mag., vol. 47, no. 4, Apr. 2009, pp. $74-81$.

8. G. Li and H. Liu, "Downlink Radio Resource Allocation for MultiCell OFDMA System," IEEE Trans. Wireless Commun., vol. 5, no. 12, Dec. 2006, pp. 3451-59.

9. D. Gesbert et al., "Adaptation, Coordination, and Distributed Resource Allocation in Interference-Limited Wireless Networks," Proc. IEEE, vol. 95, no. 12, Dec. 2007, pp. 2393-2409.
10. S. Sadr, A. Anpalagan, and K. Raahemifar, "Radio Resource Allocation Algorithms for the Downlink of Multiuser OFDM Communication Systems," IEEE Commun. Surveys \& Tutorials, vol. 11, no. 3, 3rd Qtr. 2009, pp. 92-106.

11. E. Yaacoub and Z. Dawy, "A Survey on Uplink Resource Allocation in OFDMA Wireless Networks," IEEE Commun. Surveys \& Tutorials, vol. 14, no. 2, 2nd Qtr. 2012, pp. 322-37.

12. N. Saquib et al., "Interference Management in OFDMA Femtocell Networks: Issues and Approaches," IEEE Wireless Commun., vol. 19, no. 3, June 2012, pp. 86-95.

13. E. Dahlman et al., $3 G$ Evolution: HSPA and LTE for Mobile Broadband, 2nd ed., Academic Press, 2008.

14. J. Mo, H. S. W. So, and J. Walrand, "Comparison of Multichannel MAC Protocols," IEEE Trans. Mobile Computing, vol. 7, no. 1, Jan. 2008, pp. 50-65.

15. Y.-J. Choi, S. Park, and S. Bahk, "Multichannel Random Access in OFDMA Wireless Networks," IEEE JSAC, vol. 24, no. 3, Mar. 2006, pp. 603-13.

16. H. Kwon et al., "Generalized CSMA/CA for OFDMA Systems: Protocol Design, Throughput Analysis, and Implementation Issues," IEEE Trans. Wireless Commun., vol. 8, no. 8, Aug. 2009, pp. 4176-87.

17. M. L. Chaudhry and J. Templeton, A First Course in Bulk Queues, Wiley, 1983. 